\title{
The Characteristic Property of Five Parameter Type II Generalized Logistic Distribution
}

\author{
Sule Ibrahim ${ }^{1}$, Olalekan Akanji Bello ${ }^{1}$, Awodutire Phillip Oluwatobi ${ }^{2,}$, \\ Hammed Olanrewaju Lawal ${ }^{3}$ \\ ${ }^{1}$ Department of Mathematics and Statistics, Ahmadu Bello University, Zaria, Nigeria \\ ${ }^{2}$ Department of Mathematical Science, University of Africa, Toru Orua, Nigeria \\ ${ }^{3}$ Department of Mathematics, Obafemi Awolowo University, Ile-Ife, Nigeria
}

\section{Email address:}

ibrahimsule76@yahoo.com (S. Ibrahim), olalekan4sure@gmail.com (O. A. Bello), phillip.awodutire@gmail.com (A. P. Oluwatobi), lawalikan@yahoo.com (H. O. Lawal)

${ }^{*}$ Corresponding author

\section{To cite this article:}

Sule Ibrahim, Olalekan Akanji Bello, Awodutire Phillip Oluwatobi, Hammed Olanrewaju Lawal. The Characteristic Property of Five Parameter Type II Generalized Logistic Distribution. International Journal of Statistical Distributions and Applications.

Vol. 6, No. 4, 2020, pp. 71-74. doi: 10.11648/j.ijsd.20200604.12

Received: October 29, 2026; Accepted: December 3, 2016; Published: December 25, 2020

\begin{abstract}
Order statistics are among the most fundamental tools in non-parametric statistics and inference. Special important cases of the order statistics are the minimum and maximum value of a sample, sample median and other sample quantiles. On this note, we obtained the $r^{\text {th }}$ minimum and maximum order statistic for the five parameter type II generalized logistic distribution using the probability distribution function and cumulative density function to obtain another five parameter type II generalized logistic distribution which shares the same properties by replacing $p$ with $n p$. We also obtain the quantile function by inverting the cumulative density function of the distribution which can be used to generate random samples arising from the distribution. The survival and hazard functions of the distribution are also obtained.
\end{abstract}

Keywords: Characterizations, Generalization, Hazard Function, Logistic Distribution, Order Statistics, Parameter, Reliability, Survival Function

\section{Introduction}

In statistics, the $k^{\text {th }}$ order statistic of a statistical sample is equal to its $k^{\text {th }}$ minimum value and together with rank statistics, order statistics are among the most fundamental tools in non-parametric statistics and inference. Special important cases of the order statistics are the minimum and maximum value of a sample, sample median and other sample quantiles.

When using probability theory to analyze order statistics of random samples from a continuous distribution, the cumulative distribution function (cdf) is used to reduce the analysis. Because of its simplicity and mathematical tractability, order statistics from the logistic distribution have received much attention and have been studied by several authors like Birnbaum and Dudman (1963), Gupta and Shah (1965), Tarter and Clark (1980), Shah (1966). Begum and
Parvin (2002), studied the Moments of Order Statistics from Doubly Truncated Burr Distribution. Mathai (2003), studied the Order statistics from a logistic distribution and application to survival and reliability analysis. Mohammad et al (2012), worked on the Characterizations of Logistic Distribution through Order Statistics with independent Exponential Shift. Shah (1966), studied the Bivariate Moments of Order Statistics from a Logistic Distribution. Balakrishnan and Saleh (2011), studied the Relations for moments of progressively type II censored order Statistics from Log-logistic Distribution with applications to Inference. Balakrishnan and Leung (1988), studied the Order statistics from the Type I generalized Logistic Distribution.

Orderstatistics andtheir moments have beenstudied ingreat detail by several authorsincluding Tarterand Clark (1965), 
Shah (1966, 1970), Gupta et al. (1967), Malik (1980), George and Rousseau (1987), and Balakrishnan and Malik (1990). Birnbaum and Dudman (1963) derived explicit expression for the cumulants of order statistics and tabulated the means and standard deviations for sample sizes up to ten and for some large sample sizes as well. They then summarized these quantities in graphs to facilitate interpolation to other sample sizes. Gupta and Shah (1965) derived exact expressions for the moments of order statistics in terms of Bernoulli and Stirling numbers of first kind and used them to tabulate the first four moments for sample sizes up to ten. They also expressed the cumulants in terms of polygamma functions, as was originally pointed out by Plackett (1958). It should be mentioned here that Plackett (1958) used these explicit expressions of the moments of logistic order statistics to develop a method of approximating the moments of order statistics from an arbitrary continuousdistribution. Distribution of the sample range has been studied by Gupta and Shah (1965) who also provided a short table of its percentage points for $n=2$ and 3. By generalizing this result, Malik (1980) derived the exact formula for the cumulative distribution function of the $r^{\text {th }}$ quasi-range.

The Logistic distribution is a continuous probability density function that is symmetric and unimodal. It is similar in appearance to the Normal distribution and in practical applications, the two distributions cannot be distinguished from one another. The logistic distribution has been one of the most important statistical distributions because of its simplicity and also its historical importance as a growth curve. Some applications of logistic distributions in the analysis of quantal response data, probit analysis, and dosage response studies, and in several other situations have been mentioned by Johnson, Kotz and Balakrishnan (1995). The generalized logistic distributions are very useful classes of densities as they possess a wide range of indices of skewness and kurtosis. Therefore an important application of the generalized logistic distribution is its use in studying robustness of estimators and tests. Balakrishnan and Leung (1988) present two real data examples for the usefulness of the generalized logistic distribution. One about oxygen concentration and another about resistance of automobile paint. In both examples the authors choose $b=2$ by eye and verify the validity of this assumption by Q-Q plots.

Olapade, et al., (2016) derived the five-parameter type II generalized logistic distribution by extending Olapade (2005). The derived distribution has the probability density function (pdf) as

$$
f(x)=\frac{\lambda p \beta^{p} e^{p x}}{\left(\lambda+\beta e^{x}\right)^{p+1}},-\infty<x<\infty .
$$

and cumulative density function (cdf)

$$
F(x ; \beta, \lambda, p)=1-\left[\frac{\beta e^{x}}{\lambda+\beta e^{x}}\right]^{p} .
$$

The main objective of this paper is to obtain some of the prop erties the five-parameter type II generalized logistic distributi on.

The rest of the paper is organized as follows. In section 2, we discuss the linear representation of the five parameter type II generalized logistic distribution obtained in Olapade et al (2016). Section 3 presents the quantile function. Section 4 presents the order statistic by obtaining the maximum order statistic by letting $r=n$ in the $r^{\text {th }}$ order statistic and the minimum order statistic by letting $r=1$ in the $r^{\text {th }}$ order statistic. In section 5, we discuss the survival function and in section 6 , we discuss the hazard function. Finally, we present the concluding remark in section 7 .

\section{Infinite Mixture Representation}

In this section, we derived the linear representation of the five-parameter type II generalized logistic distribution which can be used to obtain some of the properties of the distribution.

Consider following series expansion

$$
(a+y)^{n}=\sum_{i=0}^{\infty}\left(\begin{array}{c}
n \\
i
\end{array}\right) a^{n-i} y^{i}
$$

The linear representation of the five-parameter type II generalized logistic distribution in () is obtained as follows:

Consider

$$
\left(\lambda+\beta e^{x}\right)^{-p-1}=\sum_{i=0}^{\infty}\left(\begin{array}{c}
-p-1 \\
i
\end{array}\right) \lambda^{-p-1-i}\left(\beta e^{x}\right)^{i}
$$

Substituting () into (), we have

$$
\begin{gathered}
f(x ; \beta, \lambda, p)=\lambda p \beta^{p} \beta^{r} \sum_{i=0}^{\infty}\left(\begin{array}{c}
-p-1 \\
i
\end{array}\right) \lambda^{-p-1-i} e^{x(i+p)} \\
f(x ; \beta, \lambda, p)=p \beta^{p+i} \sum_{i=0}^{\infty}\left(\begin{array}{c}
-p-1 \\
i
\end{array}\right) \lambda^{-p-i} e^{x(i+p)}
\end{gathered}
$$

\section{Quantile Function of the Five-Parameter Type II Generalized Logistic Distribution}

The five parameter type II generalized logistic distribution is easily simulated by inverting (2) as follows: if $u$ has a uniform $\mathrm{U}(0,1)$ distribution, then the solution of the nonlinear equation is given by

$$
x=Q(u)=\log \left[\frac{\lambda\left(1-u^{\frac{1}{p}}\right)}{\beta u^{\frac{1}{p}}}\right]
$$

\section{Order Statistics of the Five-Parameter Type II Generalized Logistic Distribution}

Definition 1: If the random variables $\mathrm{X}_{1}, \mathrm{X}_{2}, \ldots \ldots, \mathrm{X}_{\mathrm{n}}$ are arranged in increasing order of magnitude as $X_{(1)} \leq X_{(2)}$ $\leq \ldots \ldots \ldots . \leq X_{(n)} . X_{(r)}$ is called the $r^{\text {th }}$ order statistics $(\mathrm{r}=1,2, \ldots \mathrm{n})$. The unordered $X_{r}, \mathrm{r}=1,2, \ldots, \mathrm{n}$ are assumed to be statistically 
independent and identically distributed. The subject of order statistics deals with the distribution of the $r^{\text {th }}$ order statistics and applications of these ordered random variables.

Definition 2: Let $X_{1}, X_{2}, \ldots, X_{n}$ be $\mathrm{n}$ independently continuous random variable from the five parameter type II generalized logistic distribution and let $X_{(1)} \leq X_{(2)} \leq \ldots \leq X_{(n)}$ be the corresponding order statistics.

Let $F_{X r: n}(x)$ and $f_{X r: n}(x), \mathrm{r}=1,2,3, \ldots, \mathrm{n}$ denote the cumulative distribution function and the probability density function of the $r^{\text {th }}$ order statistics $x_{r: n}$ respectively. David (1970) gave the probability density function of $x_{r: n}$ as

$$
f_{X_{r n}}=\frac{1}{B(r, n-r+1)} F^{r-1}(x)[1-F(x)]^{n-r} f(x)
$$

By substituting equations (1) and (2) into equation (8) we have

$$
\begin{gathered}
X_{r-n}(x)=\frac{1}{B(r, n-r+1)}\left[1-\frac{\beta^{p} e^{p x}}{\left(\lambda+\beta e^{x}\right)^{p}}\right]^{r-1}\left[\frac{\beta^{p} e^{p x}}{\left(\lambda+\beta e^{x}\right)^{p}}\right]^{n-r} \frac{\lambda p \beta^{p} e^{p x}}{\left(\lambda+\beta e^{x}\right)^{p+1}} \\
f_{X_{r n n}}(x)=\frac{\lambda p \beta^{p} e^{p x}}{B(r, n-r+1)\left(\lambda+\beta e^{x}\right)^{p+1}}\left[1-\frac{\beta^{p} e^{p x}}{\left(\lambda+\beta e^{x}\right)^{p}}\right]^{r-1}\left[\frac{\beta^{p} e^{p x}}{\left(\lambda+\beta e^{x}\right)^{p}}\right]^{n-r}
\end{gathered}
$$

The equation (10) is called the pdf of the $r^{\text {th }}$ order statistics for the five parameter type II generalized logistic distribution.

\subsection{The Maximum Order Statistics from the Five Parameter Type II Generalized Logistic Distribution}

Consider the probability density of the $r^{\text {th }}$ order statistics from the five parameter type II generalized logistic distribution in equation (5). Let $\mathrm{r}=\mathrm{n}$, then the probability density function of the maximum order statistic is

$$
\begin{aligned}
& f_{X_{n n}}(x)=\frac{\lambda p \beta^{p} e^{p x}}{B(n, 1)\left(\lambda+\beta e^{x}\right)^{p+1}}\left[1-\frac{\beta^{p} e^{p x}}{\left(\lambda+\beta e^{x}\right)^{p}}\right]^{n-1} \\
& f_{X_{n n}}(x)=\frac{\lambda n p \beta^{p} e^{p x}}{\left(\lambda+\beta e^{x}\right)^{n p+1}}\left[\left(\lambda+\beta e^{x}\right)^{p}-\beta^{p} e^{p x}\right]^{n-1}
\end{aligned}
$$

Using Binomial expansion, we have

$$
f_{X_{n: n}}(x)=n p \sum_{k=0}^{n-1} \sum_{j=0}^{p k-n p-1}(-1)^{n-1-k}\left(\begin{array}{l}
n-1 \\
k
\end{array}\right)\left(\begin{array}{l}
p k-n p-1 \\
j
\end{array}\right) \beta^{n p-p k+j} \lambda^{p k-n p-j} x^{p} e^{x(n p-p k+j}
$$

The $p^{\text {th }}$ moment of the maximum order statistics is obtained as

$$
E\left[x_{n: n}^{p}\right]=n p \sum_{k=0}^{n-1} \sum_{j=0}^{p k-n p-1}(-1)^{n-1-k}\left(\begin{array}{l}
n-1 \\
k
\end{array}\right)\left(\begin{array}{l}
p k-n p-1 \\
j
\end{array}\right) \beta^{n p-p k+j} \lambda^{p k-n p-j} \int_{-\infty}^{\infty} x^{p} e^{x(n p-p k+j)} d x
$$

\subsection{The Minimum Order Statistics from the Five Parameter Type II Generalized Logistic Distribution}

Consider the probability density of the $r^{\text {th }}$ order statistics from the five parameter type II generalized logistic distribution in equation (5). To obtain the distribution function of the minimum order statistics of type II generalized logistic distribution, we consider equation (5) and make $r=1$. Then we have

$$
\begin{gathered}
f_{x_{1: n}}(x)=\frac{\lambda p \beta^{p} e^{p x}}{B(1, n)\left(\lambda+\beta e^{x}\right)^{p+1}}\left[\frac{\beta^{p} e^{p x}}{\left(\lambda+\beta e^{x}\right)^{p}}\right]^{n-1} \\
f_{x_{1 n}}(x)=\frac{\lambda p}{B(1, n)}\left[\frac{\beta^{n p} e^{n p x}}{\left(\lambda+\beta e^{x}\right)^{n p+1}}\right]^{n-1}
\end{gathered}
$$

$$
f_{x_{1: n}}(x)=\frac{n p \lambda \beta^{n p} e^{p x}}{\left(\lambda+\beta e^{x}\right)^{n p+1}}
$$

The equation (17) is another five parameter type II generalized logistic distribution with parameters $\lambda, \beta$ and $n p$. The distribution also shares the same properties with the five parameter type II generalized logistic distribution derived in Olapade et al (2016) by replacing parameter $p$ with parameter $n p$

\section{Survival Function}

The survival function is also known as reliability function, is the probability of an item not failing prior to some time. It can be defined as 


$$
\begin{aligned}
& S(x)=1-F(x) \\
& S(x)=\left[\frac{\beta e^{x}}{\lambda+\beta e^{x}}\right]^{p}
\end{aligned}
$$

\section{Hazard Function}

The hazard rate function is given as

$$
\begin{gathered}
H(x)=\frac{f(x)}{1-F(x)}=\frac{f(x)}{S(x)} \\
H(x)=\frac{\frac{\lambda p \beta e^{p}}{\left(\lambda+\beta e^{x}\right)^{p+1}}}{\left[\frac{\beta e^{x}}{\lambda+\beta e^{x}}\right]^{p}} \\
H(x)=\frac{\lambda p}{\left(\lambda+\beta e^{x}\right)^{p}}
\end{gathered}
$$

\section{Conclusion}

This paper presented a new distribution using the order statistic. The new distribution shares the same properties with the pdf of Olapade et al (2016) by replacing parameter $p$ with $\mathrm{np}$. Some properties of the five parameter type II generalized logistic distribution were studied. Further research work can be done on estimation methods and real-life application.

\section{References}

[1] Balakrishnan, N. and Leung, M. Y. (1988), Order statistics from the Type I generalized Logistic Distribution, Communications in Statistics - Simulation and Computation. Vol. 17 (1), 25-50.

[2] Balakrishnan, N. and Kocherlakota, S. (1986). On the moments of order statistics from the doubly truncated logistic distribution, Journal of Statistical Planning and Inference. 13, 117-129.

[3] Balakrishnan, N. and Saleh, H. M. (2011), Relations for moments of progressively Type II censored order Statistics from Log-logistic Distribution with applications to Inference, Computational Statistics and Data Analysis, 55 (10), pp 27752792.

[4] Begum, A. A. and Parvin, S. (2002), Moments of Order Statistics from Doubly Truncated Burr Distribution, J. Statist. Research, 36 (2), 179-190.

[5] Birnbaum, A. and Dudman, J. (1963), Logistic Order statistics, Ann. Math. Statist., 34 (2), pp 658-663.
[6] David, H. A. (1970), Order Statistics. John Wiley, New York.

[7] George, E. 0. and Rousseau, C. C. (1987). On the logistic midrange, Annals of the Institute Statistical Mathematics. 39, 627-635.

[8] Gupta, S. S. and Shah, B. K. (1965), Exact Moments and Percentage Points of the Order Statistics and the Distribution of the Range from the Logistic Distribution, Annals of Mathematical Statistics, 36 (3), pp 907-920.

[9] Govindarajulu, Z. (1963), On moments of order statistics and quasi-ranges from normalpopulations, Annals of Mathematical Statistics. 34, 633-651.

[10] Johnson, N. L., Kotz S., Balakrishnan N. (1995), Continuous Univariate Distributions Volume 2. Wiley, New York.

[11] Mathai, A. M. (2003), Order statistics from a logistic distribution and applications to survival and reliability analysis, IEEE Transactions on Reliability, 52 (2), pp 200-206.

[12] Mohammad, A., George, P. Y and Contantic, O. (2012), Characterizations of Logistic Distribution through Order Statistics with independent Exponential Shift, Economic Quality Control, 27 (1), pp 85-96.

[13] Olapade, A. K., Sule, I., Bello, A. O. and Braimah, O. J. (2016), On a Five Parameter Type II Generalized Logistic Distribution, Computing, Information Systems, Development Informatics and Allied Research Journal, 7 (1), pp 49-58.

[14] Olapade, A. K. (2000), Some properties of the Type I Generalized logistic distribution. Intro. Stat. 2.

[15] Plackett, R. L. (1958). Linear estimation from censored data, Annals of Mathematical Statistics, 29, 131-142.

[16] Shah, B. K. (1966), On the Bivariate Moments of Order Statistics from a Logistic Distribution, The Annals of Mathematical Statistics, 37 (4), 1002-1010.

[17] Shah, B. K. (1970). Note on moments of a logistic order statistics, Annals of Mathematical Statistics. 41, 2151-2152.

[18] Tarter, M. E. and Clark, V. A. (1980), Correction to "Order Statistics of Logistic Variates", Annals of Mathematical Statistics, 8 (4), pp-935.

[19] Tarter, M. E. (1966). Exact moment sand product moments of the order statistics from the truncated logistic distribution, Journal of American Statistical Association, 61, 514-525.

[20] Tarter, M. E. and Clark, V. A. (1965). Properties of the median and other order statistics of logistic variates, Annals of Mathematical Statistics, 36, 1779-1786. 